\title{
Hybrid Tamm-cavity modes in photonic crystal with resonant nanocomposite defect layer
}

\author{
S.Ya. Vetrov ${ }^{1,2}$, A.Yu. Avdeeva ${ }^{2}$, M.V. Pyatnov ${ }^{2,1}$, I.V. Timofeev ${ }^{2,1}$ \\ ${ }^{1}$ Siberian Federal University, 660041, Russia, Krasnoyarsk, pr. Svobodny 79, \\ ${ }^{2}$ Kirensky Institute of Physics, Federal Research Center KSC SB RAS, \\ 660036, Russia, Krasnoyarsk, Akademgorodok 50/38
}

\begin{abstract}
Hybrid optical modes in a one-dimensional photonic crystal with a resonant nanocomposite defect bounded by a metallic layer are studied. The nanocomposite consists of spherical metallic constituents, that are distributed in a dielectric matrix. Transmittance, reflectance, and absorbance spectra of this structure, which is shined by light with normal incidence, are calculated. The possibility of control of the hybrid modes spectral characteristics by changing the thickness of the layer adjacent to the metal, the number of layers, and the nanocomposite filling factor is shown.

Keywords: photonic crystals, nanocomposite, microcavities, localized modes.

Citation: Vetrov SYa, Avdeeva AYu, Pyatnov MV, Timofeev IV. Hybrid Tamm-cavity modes in photonic crystal with resonant nanocomposite defect layer. Computer Optics 2020; 44(3): 319324. DOI: 10.18287/2412-6179-CO-637.

Acknowledgements: This research was funded by the Russian Foundation for Basic Research, Government of Krasnoyarsk Territory, Krasnoyarsk Region Science and Technology Support Fund to the research Project No 18-42-243025.
\end{abstract}

\section{Introduction}

A Tamm plasmon (TP) is a localized surface mode that can be excited even under normal incidence of light of any polarization [1-4]. Such a mode is supported by the interface between a metallic layer and a photonic band-gap material, i. e., a photonic crystal (PC). The TP can be experimentally observed as a narrow peak in the reflectance or transmittance spectrum of a sample. In the localized modes of this type, the field decays exponentially inside both media with an increase in the distance from the interface. The decay deep in the metallic layer is caused by the negative permittivity of a metal and the decay deep in the photonic band-gap material, by the Bragg reflection.

Various ways of exciting the hybrid modes formed by the TP and resonances of different nature (surface plasmons, exciton modes, etc.) have been developed [5-11]. The interest in these interactions is due to the potential of their application in sensors $[12,13]$, light-emitting devices [14, 15], photodiodes [16], and organic solar cells [17]. The variant of hybridization of the TP and cavity modes (CMs) is the most widespread. In this case, the electromagnetic field energy is localized simultaneously on a structural defect and a $\mathrm{PC} /$ metallic film interface. The change in the parameters of a structure, including the refractive index or thickness of the layer adjacent to the metal, leads to the change in the bonding value, which is followed by the reconstruction of the spectrum [18]. The hybridization makes it possible to enhance the field in a microcavity and reduce the absorption in metallic layers embedded in a structure [19].

New possibilities for forming hybrid modes (HMs) and controlling the spectral properties of a PC are opened by using metal-dielectric nanocomposites (NCs) with the resonant dispersion [20-26]. The position and width of the $\mathrm{NC}$ resonance region lying within the visible spectral range depends on the permittivities of initial materials and concentration, size, and shape of nanoparticles. The effective characteristics of an NC consisting of metallic nanoparticles dispersed in a dielectric matrix are formed due to the plasmon resonance of nanoparticles and, in the optical range, can take unique values atypical of natural materials; for instance, the real part of the effective refractive index can be much more than unity, similar to unity, or less than unity and take a zero value as well [27-29].

The aim of this study is to investigate the hybrid optical modes caused by the bonding between the cavity modes of a PC with a resonant $\mathrm{NC}$ defect and the optical $\mathrm{TP}$ at the interface between a PC and a metallic film. We show that the Tamm and cavity components of the hybrid state manifest themselves in the transmittance spectrum as repulsing resonances. We examine the dependence of the repulsion of the hybrid state components in the transmittance spectrum on the thickness of the PC layer adjacent to the metallic film. It is established that the position of the hybrid modes in the PC band gap is sensitive to the change in the nanoparticle concentration in an NC.

\section{System description and methods for determing the transmittance}

The model to investigate is a one-dimensional PC bounded by a 50 -nm-thick silver film from its one side (fig. 1). The materials of alternating PC layers are zirconium dioxide $\mathrm{ZrO}_{2}$ with a permittivity of $\varepsilon_{a}=4.16$ and silicon dioxide $\mathrm{SiO}_{2}$ with a permittivity of $\varepsilon_{b}=2.10$; the layer thicknesses were 40 and $90 \mathrm{~nm}$, respectively. The PC consists of $N=23$ layers; the center $\mathrm{SiO}_{2}$ layer is replaced 
by an $\mathrm{NC}$ defect layer. The thickness of the $\mathrm{ZrO}_{2}$ layer adjacent to the metallic film is different from $40 \mathrm{~nm}$ and specified by parameter $d$. The refractive index of the environment was assumed to be unity (air). The defect is a 160-nm-thick metal-dielectric NC layer consisting of nanoballs dispersed in transparent optical glass with a permittivity of $\varepsilon_{d}=2.56$. The choice of $\mathrm{ZrO}_{2}$ and $\mathrm{SiO}_{2}$ layers is explained by availability of these materials. Moreover, sufficiently large contrast of the refractive indices of such layers ensures formation of a wide band gap in PC as shown in [30] both experimentally and theoretically.

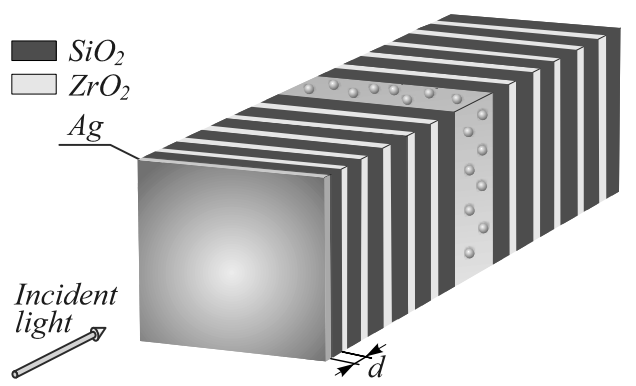

Fig. 1. Schematic of the structure

The effective permittivity [21] $\varepsilon_{m i x}$ of the NC layer is determined from the Maxwell Garnett formula widely used to study matrix media with a small volume fraction of isolated inclusions dispersed in a matrix material [22]:

$$
\varepsilon_{\text {mix }}=\varepsilon_{d}\left[1+\frac{f}{(1-f) / 3+\varepsilon_{d} /\left(\varepsilon_{m}-\varepsilon_{d}\right)}\right],
$$

here, $f$ is the filling factor (the volume fraction of nanoparticles in the matrix), $\varepsilon_{m}$ is the permittivity of the nanoparticle metal, and $\omega$ is the radiation frequency. The parameter $\varepsilon_{m}$ was specified in the form of the Drude approximation

$$
\varepsilon_{m}=\varepsilon_{0}-\frac{\omega_{p}^{2}}{\omega(\omega-i \gamma)},
$$

where $\varepsilon_{0}=5$ is the ionic core contribution, $\hbar \omega_{p}=9 \mathrm{eV}$ is the plasma frequency and $\hbar \gamma=0.02 \mathrm{eV}$ is the reciprocal relaxation time. These parameters are typical of silver [31]. The predictions of the effective Maxwell Garnett media model are valid for a moderate fraction of inclusions with a filling factor of $f<<1$. This model suggests the quasi-static approximation with the condition of a small size of nanoparticles and distance between them as compared with the optical wavelength in a medium.

Figure 2 shows dispersion dependence of the nanocomposite permittivity for two different filling factors: $f=0.01,0.06$. It can be seen that the frequency corresponding to the resonance in the defect layer shifts towards longer wavelengths with an increase in the nanoball concentration. Note that the resonant curve half-width $\varepsilon^{\prime \prime}{ }_{\text {mix }}$ is affected very little, whereas the $\varepsilon_{\text {mix }}^{\prime}$ curve is essentially modified and the range of frequencies increases for which the $\mathrm{NC}$ is similar to metal when $\varepsilon_{\text {mix }}^{\prime}<0$.

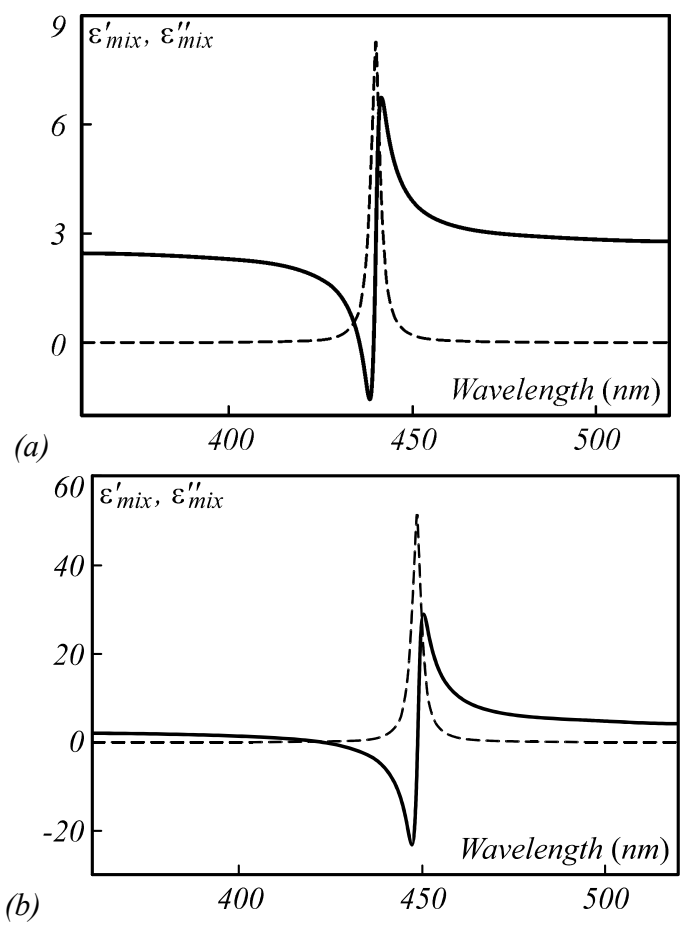

Fig. 2. Imaginary $\varepsilon^{\prime \prime}{ }_{\text {mix }}$ (dashed curve) and real $\varepsilon_{\text {mix }}^{\prime}$ (solid curve) parts of the effective permittivity \&mix versus wavelength. The filling factors $f=0.01$ (a) and $f=0.06$ (b)

The transmission of a plane light wave polarized along the $\mathrm{x}$ axis and propagating in the $z$ direction was calculated by the transfer matrix method [32]. The lightfield variation during passage through each structural layer is determined using the second-order transfer matrix and the transfer matrix of the entire structure, which relates the amplitudes of the input and output waves, is a product of such $2 \times 2$ matrices:

$$
M=T_{01} T_{02} \ldots T_{N-1, N} T_{N S},
$$

where an individual transfer matrix has the form

$$
T_{n-1, n}=\frac{1}{2}\left(\begin{array}{ll}
(1+h) e^{-i \alpha_{n} \gamma_{n}} & (1-h) e^{i \alpha_{n} \gamma_{n}} \\
(1-h) e^{-i \alpha_{n} \gamma_{n}} & (1+h) e^{i \alpha_{n} \gamma_{n}}
\end{array}\right),
$$

here, $h=\sqrt{\varepsilon_{n} / \varepsilon_{n-1}}$ is the permittivity of the $n$ layer; $\alpha_{n}=(\omega / c) \sqrt{\varepsilon(n)}$ is the frequency of the wave; $c$ is the speed of light; $\gamma_{n}=z_{n}-z_{n-1} ; n=1,2, \ldots, N ; z_{n}$ is the coordinate of the bound between the $n$ and $n+1$ layers; and $\gamma_{N+1}=0$.

The transfer matrix for the wave with orthogonal polarization is obtained using equation (4) by changing $h$ to

$$
h^{\prime}=\sqrt{\frac{\varepsilon_{n-1}}{\varepsilon_{n}}} .
$$

The transmittance, reflectance, and absorbance are 


$$
\begin{aligned}
& T(\omega)=\frac{1}{\left|\hat{M}_{11}\right|^{2}}, R(\omega)=\frac{\left|\hat{M}_{21}\right|^{2}}{\left|\hat{M}_{11}\right|^{2}}, \\
& A(\omega)=1-T(\omega)-R(\omega),
\end{aligned}
$$

here $\hat{M}_{11}$ and $\hat{M}_{21}$ - are the elements of the matrix $\hat{M}$.

\section{Results and discussion}

The parameters of the PC and metal-dielectric defect layer were chosen such that the defect mode frequency coincided with the $\mathrm{NC}$ resonant frequency. In the absence of inclusions $(f=0)$, a peak corresponding to the defect mode arises in the PC transmittance spectrum (see the inset in fig. $3 a$ ). At $f=0.01$, the $\mathrm{CM}$ is split and two peaks at wavelengths of $\lambda=419.5$ and $453 \mathrm{~nm}$ form (dashed line in fig. 3).

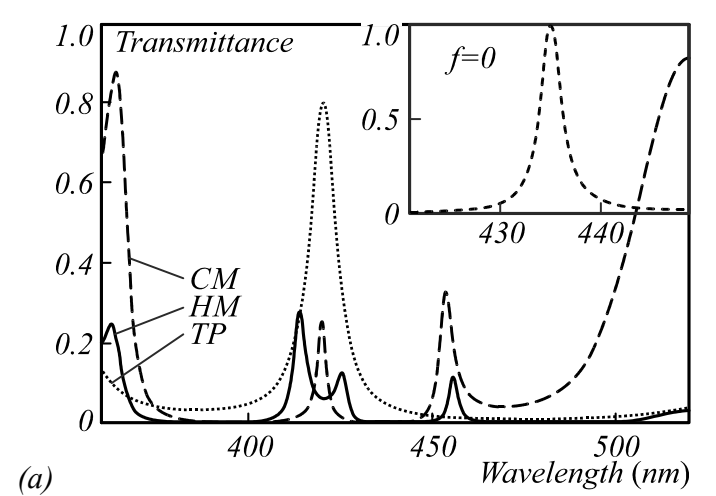

(a)
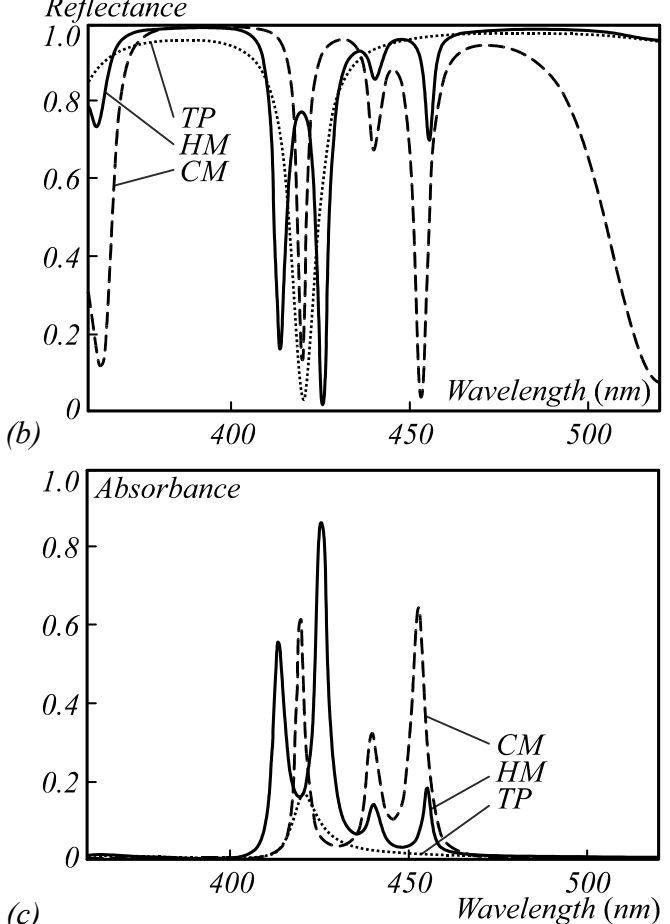

Fig. 3. (a) Transmittance, (b) reflectance, and (c) absorbance spectra. The line of dots corresponds to the structure without

a metallic layer; the dotted line, to the structure without

microcavity; and the solid line, to the entire structure.

$d=19 \mathrm{~nm}$ and $f=0.01, N=23$. Inset: transmittance spectrum of the $P C$ without metallic layer at $f=0$

With an increase in the $f$ value, the splitting grows. The splitting is caused by the change in the Fabry-Perot resonance condition due to the dispersion of the NC permittivity $\varepsilon_{m i x}$. The transmission spectrum contains also a peak at a wavelength of $440 \mathrm{~nm}$. The occurrence of this peak is consistent with the predictions following from the Fabry-Perot resonance condition [24]. It should be noted that with an increase in the $f$ value, this peak slightly shifts to the long-wavelength region ( $7 \mathrm{~nm}$ at $f=0.1$ ) with increasing absorption. This peak does not arise in the transmittance spectrum due to the high absorption.

In a PC bounded by a metallic film, another resonance type-the Tamm plasmon - can be excited at the interface. Thus, in the structure under study (fig. 1), the localized modes of two types can be excited: the TP and defect (cavity) modes. The change in the PC parameters can lead to the interaction between localized modes and formation of hybrid Tamm cavity modes.

The TP position is conventionally tuned by varying the Bragg reflector layer adjacent to the metallic layer. At a first $\mathrm{ZrO}_{2}$ layer thickness of $d=19 \mathrm{~nm}$, the spectrally pure TP (line of dots in fig. 3) and the short-wavelength cavity mode manifest themselves at the same wavelength $\lambda=419.5 \mathrm{~nm}$. The interaction between the resonances leads to the formation of two peaks at wavelengths of 413.5 and $425.5 \mathrm{~nm}$ (the black solid line in fig. 3). The splitting can be increased via increasing the spatial overlap of the modes. To do this, it is necessary to decrease the number of PC layers between the silver film and defect layer. In addition, the splitting increases with an increase in the filling factor $f$ [24].

Figure 4 shows the squared electric-field profiles of the cavity modes in the absence of a metallic layer (fig. 4a), TP in the absence of a microcavity (fig. 4b), and the hybrid Tamm cavity modes (fig. 5). For the CM, the electromagnetic field has its maximum right at the defect layer center. The TP field is localized at the metallic field. The field of the HM is localized with the maxima at the metal / PC interface and in the defect layer.

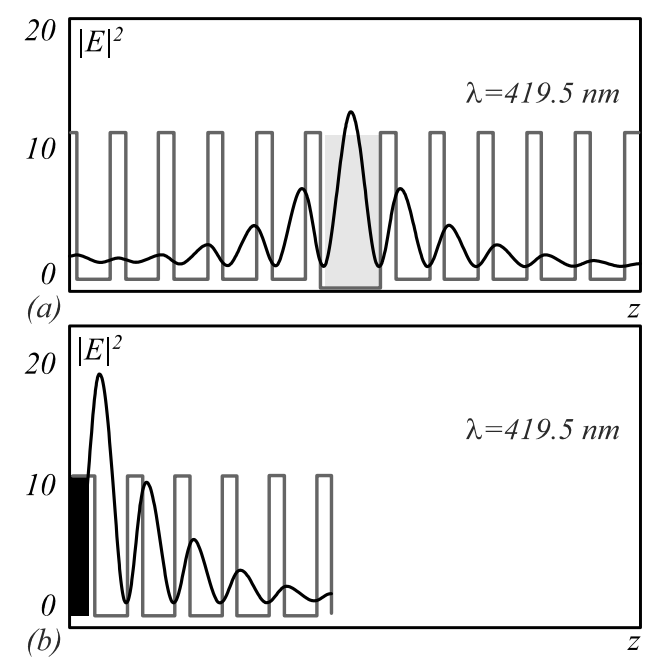

Fig. 4. Spatial distribution of the squared electric field strength for the $C M(a), T P(b) ; d=19 \mathrm{~nm}$ and $f=0.01$ 
The change in the thickness $d$ of the first layer leads to the change in the phase of the PC reflectance. Since the condition of phase matching between the reflectances from the metallic and Bragg mirrors must be met, the TP position changes. The position of the $\mathrm{CM}$ is almost insensitive to an increase in the thickness $d$, while the pure TP mode shifts and passes through the entire PC band gap.

Figure 6 shows the reflectance spectrum of the investigated structure at different $d$ values for NC filling factors of $f=0.01$ and $f=0.06$. As the d value increases from 0 to $50 \mathrm{~nm}$, the sequential hybridization occurs with all the cavity modes. This interaction manifests itself as a peak quasi-crossing in the transmittance or reflectance spectrum. At $f=0.01$, three quasi-crossings are observed. At $f=0.06$, there are four quasi-crossings and the peaks are observed at wavelengths of $\lambda=396$ and $478 \mathrm{~nm}$, as well as 444 and $457 \mathrm{~nm}$. The additional peak at a wavelength of $457 \mathrm{~nm}$ in the spectrum occurs due to the NC permittivity dispersion, which depends on the $f$ value.

The change in the number of PC periods leads to the change in the overlap of electromagnetic fields of the localized modes (fig. 7).

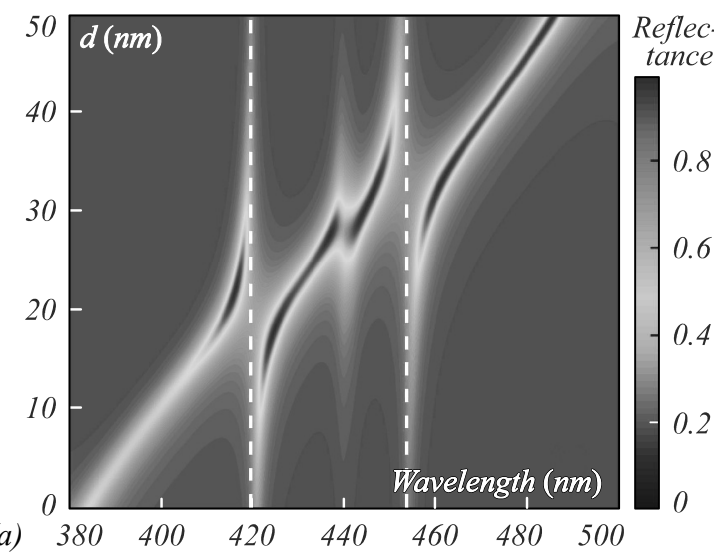

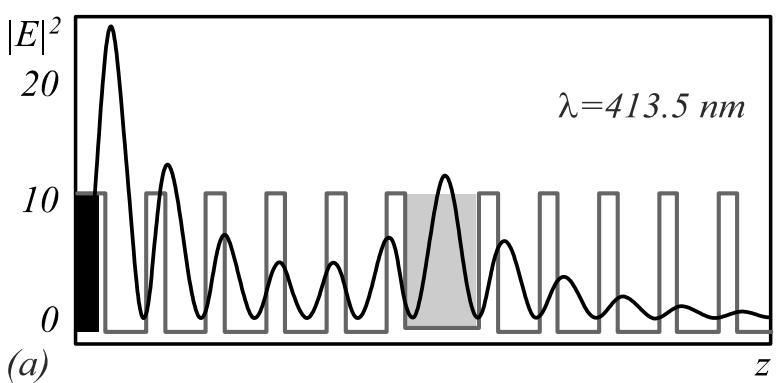

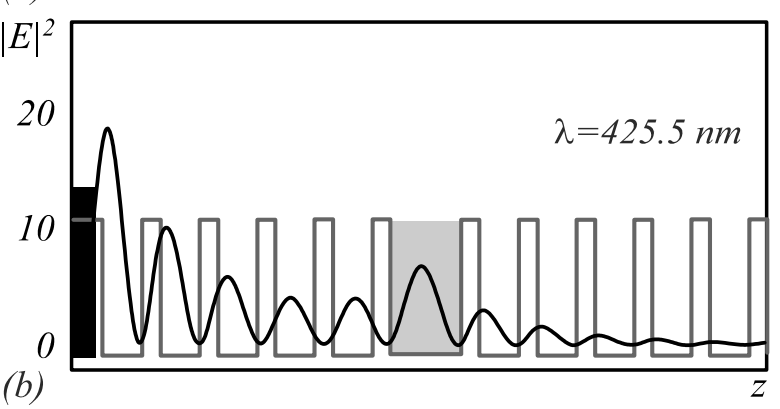

Fig. 5. Spatial distribution of the squared electric field strength for hybrid Tamm cavity modes of $413.5 \mathrm{~nm}$ (a) and $425.5 \mathrm{~nm}(b) ; d=19 \mathrm{~nm}$ and $f=0.01, N=23$

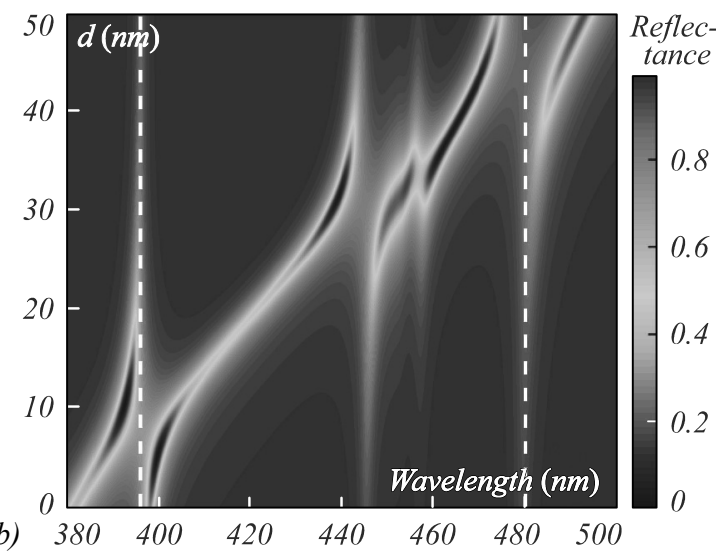

Fig. 6. Reflectance spectrum of the structure (fig. 1) $\mathrm{N}=23 \mathrm{vs}$ thickness $d$ of the $\mathrm{ZrO}_{2}$ layer adjacent to the 50-nm-thick metallic film at $f=0.01$ (a) and $f=0.06$ (b). White dashed lines show the positions of resonance-split NC cavity modes; $\lambda=419.5$ and $453 \mathrm{~nm}(\mathrm{a})$; $\lambda=396$ and $478 \mathrm{~nm}(\mathrm{~b})$

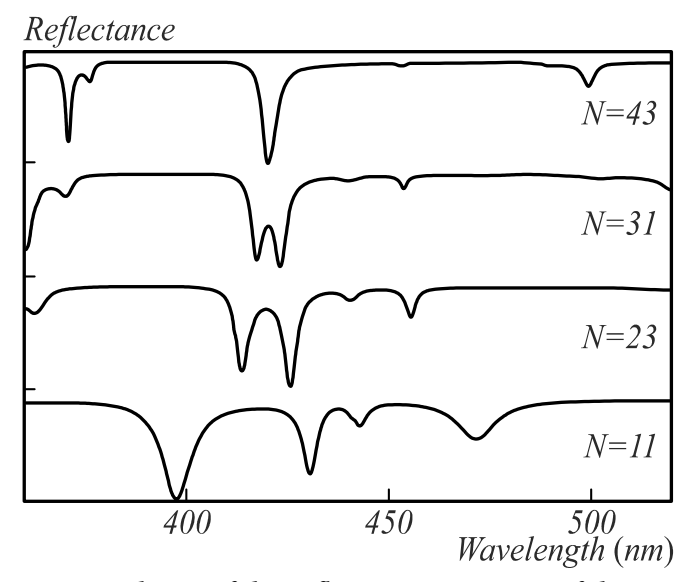

Fig. 7. Dependence of the reflectance spectrum of the structure (fig. 1) on the numbers $N$ of PC layers. In all the cases, the defect is localized at the center of the structure; $d=19 \mathrm{~nm}, f=0.01$
A decrease in the distance between the metallic layer and defect leads to an increase in the peak splitting. With an increase in the number of PC periods, the CM and TP peak wavelengths degenerate. With an increase in the filling factor $f$ and other parameters unchanged, the reflectance spectrum (fig. 7) changes significantly. The more splitting increases, the more degree of mode hybridization decreases.

\section{Conclusions}

Thus, we demonstrated the existence of hybrid optical modes caused by the bonding between the cavity modes of the Bragg reflector with a nanocomposite defect and the Tamm plasmon excited at the interface between a PC and a thin metallic film. We showed the possibility for controlling the spectral properties via changing the thickness of a layer adjacent to the metal and a number of PC layers, which can be used to create a tunable filter on the 
basis of the proposed structure. The spectral properties can be additionally governed by changing the nanocomposite filling factor. The sensitivity of the position of split peaks in the transmittance spectrum to the parameters of the structure can be used to govern light in devices based on such structures.

\section{Acknowledgements}

This research was funded by the Russian Foundation for Basic Research, Government of Krasnoyarsk Territory, Krasnoyarsk Region Science and Technology Support Fund to the research Project No 18-42-243025.

\section{References}

[1] Goto T, Dorofeenko AV, Merzlikin AM, Baryshev AV, Vinogradov AP, Inoue M, Lisyansky AA, Granovsky AB. Optical Tamm states in one-dimensional magnetophotonic structures. Phys Rev Lett 2008; 101: 113902.

[2] Kaliteevski MA, Iorsh I, Brand S, Abram RA, Chamberlain JM, Kavokin AV, Shelykh IA. Phys Rev B 2007; 76: 165415.

[3] Afinogenov BI, Bessonov VO, Soboleva IV and Fedyanin AA. Ultrafast all-optical light control with Tamm plasmons in photonic nanostructures. ACS Photon 2019; 6: 844-850.

[4] Cheng H-C, Kuo C-Y, Hung Y-J, Chen K-P, Jeng S-C. Liquid-crystal active Tamm-plasmon devices. Phys Rev Appl 2018; 9: 064034.

[5] Kaliteevski M, Brand S, Abram RA, Iorsh I, Kavokin AV and Shelykh IA. Hybrid states of Tamm plasmons and exciton polaritons. Appl Phys Lett 2009; 95: 251108.

[6] Pankin PS, Vetrov SYa, Timofeev IV. Tunable hybrid Tamm-microcavity states. J Opt Soc Am B 2017; 34: 2633-2639.

[7] Pyatnov MV, Vetrov SY, Timofeev IV. Tunable hybrid optical modes in a bounded cholesteric liquid crystal with a twist defect. Phys Rev E 2018; 97: 032703.

[8] Afinogenov BI, Bessonov VO, Nikulin AA, Fedyanin AA. Observation of hybrid state of Tamm and surface plasmonpolaritons in one-dimensional photonic crystals. Appl Phys Lett 2013; 103: 061112.

[9] Paulauskas A, Tumenas S, Selskis A, Tolenis T, Valavicius A, Balevicius Z. Hybrid Tamm-surface plasmon polaritons mode for detection of mercury adsorption on 1D photonic crystal/gold nanostructures by total internal reflection ellipsometry. Opt Express 2018; 26: 30400-30408.

[10] Wurdack M, Lundt N, Klaas M, Baumann V, Kavokin AV, Höfling S, Schneider C. Observation of hybrid Tammplasmon exciton-polaritons with GaAs quantum wells and a MoSe 2 monolayer. Nat Commun 2017; 8: 259.

[11] Khokhlov NE, Prokopov AR, Shaposhnikov AN, Berzhansky VN, Kozhaev MA, Andreev SN, Ravishankar AP, Achanta VG, Bykov DA, Zvezdin AK, Belotelov VI. Photonic crystals with plasmonic patterns: novel type of the heterostructures for enhanced magneto-optical activity. J Phys D Appl Phys 2015; 48: 095001. DOI: 10.1088/0022-3727/48/9/095001.

[12] Maji PS, Shukla MK, Das R. Blood component detection based on miniaturized self-referenced hybrid Tamm-plasmonpolariton sensor. Sens Actuators B Chem 2018; 255: 729-734.
[13] Zhang X, Zhu XS, Shi YW. An optical fiber refractive index sensor based on the hybrid mode of Tamm and surface plasmon Polaritons. Sensor 2018; 18: 2129.

[14] Zhang X-L, Feng J, Han X-C, Liu Y-F, Chen Q-D, Song JF, Sun H-B. Hybrid Tamm plasmon-polariton/microcavity modes for white top-emitting organic light-emitting devices. Optica 2015; 2: 579-584.

[15] Geng D, Cabello-Olmo E, Lozano G, Míguez H. Tamm plasmons directionally enhance rare-earth nanophosphor emission. ACS Photon 2019; 6: 634-641.

[16] Mischok A, Siegmund B, Ghosh DS, Benduhn J, Spoltore D, Böhm M, Fröb H, Körner C, Leo K, Vandewal K. Controlling Tamm plasmons for organic narrowband nearinfrared photodetectors. ACS Photon 2017; 4: 2228-2234.

[17] Zhang X-L, Song J-F, Feng J, Sun H-B. Spectral engineering by flexible tunings of optical tamm states and Fabry-Perot cavity resonance. Opt Lett 2013; 38: 4382.

[18] Rahman SKS-U, Klein T, Gutowski J, Klembt S, Sebald $\mathrm{K}$. Tunable Bragg polaritons and nonlinear emission from a hybrid metal-unfolded ZnSe-based microcavity. Sci Rep 2017; 7: 767.

[19] Kaliteevcki MA, Lazarenko AA, Ilinskaya ND, M. Zadiranov YuM, Sasin ME, Zaitsev D, Mazlin VA, Brunkov PN, Pavlov SI, Egorov AYu. Experimental demonstration of reduced light absorption by intracavity metallic layers in Tamm plasmon-based microcavity. plasmonics 2015; 38: 281-284.

[20] Atwater HA, Polman A. Plasmonics for improved photovoltaic devices. Nat Mater 2010; 9: 205-213.

[21] Sihvola A. Electromagnetic mixing formulae and applications. London: The Institution of Electrical Engineers; 1999.

[22] Markel VA. Introduction to the Maxwell Garnett approximation: tutorial. J Opt Soc Am A 2016; 33: 12441256.

[23] Husaini S, Deych L, Menon VM. Plasmon-resonanceinduced enhancement of the reflection band in a onedimensional metal nanocomposite photonic crystal. Opt Lett 2011; 36: 1368-1370.

[24] Vetrov SYa, Avdeeva AYu, Timofeev IV. Spectral properties of a one-dimensional photonic crystal with a resonant defect nanocomposite layer. J Exp Theor Phys 2011; 113: 755-761.

[25] Aly AH, Elsayed HA, Malek C. Optical properties of onedimensional defective photonic crystal containing nanocomposite material. J Nonlinear Opt Phys 2017; 26(01): 1750007.

[26] Moiseev SG, Ostatochnikov VA. Defect modes of onedimensional photonic-crystal structure with a resonance nanocomposite layer. Quant Electron 2016: 46(8): 743-748.

[27] Oraevskii AN, Protsenko IE. Optical properties of heterogeneous media. Quant Electron 2001; 31: 252-256.

[28] Sukhov SV. Nanocomposite material with the unit refractive index. Quant Electron 2005; 35: 741-744.

[29] Moiseev SG. Active Maxwell-Garnett composite with the unit refractive index. Physica B 2010; 405: 3042-3045.

[30] Arkhipkin VG, Gunyakov VA, Myslivets SA, Gerasimov VP, Zyryanov VYa, Shabanov VF, Vetrov SYa. One-dimensional photonic crystals with a planar oriented nematic layer: Temperature and angular dependence of the spectra of defect modes. J Exp Theor Phys 2008; 106(2): 388-398.

[31] Klimov V. Nanoplasmonics. Boca Raton, FL: Pan Stanford Publishing; 2014

[32] Yeh PJ. Electromagnetic propagation in birefringent layered media. J Opt Soc Am 1979; 69: 742-756. 


\section{Authors' information}

Stepan Yakovlevich Vetrov (b. 1946), Doctor of Physico-Mathematical sciences, Professor of Theoretical Physics and Wave Phenomena department of Siberian Federal University, Senior Researcher in Laboratory of Molecular Spectroscopy, Kirensky Institute of Physics, Federal Research Center KSC SB RAS. Research interests: photonic crystals, liquid crystals, nanophotonics. E-mail: $\underline{\text { S.Vetrov@inbox.ru . }}$

Anastasia Yurievna Avdeeva (b. 1986), Junior Researcher in Laboratory for Photonics of Molecular Systems, Kirensky Institute of Physics, Federal Research Center KSC SB RAS. Research interests: photonic crystals, liquid crystals, nanocomposites. E-mail: avdeeva.anastasia@gmail.com .

Maxim Vladimirovich Pyatnov (b. 1992), PhD in Physico-mathematical sciences, Researcher in Laboratory for Photonics of Molecular Systems, Kirensky Institute of Physics, Federal Research Center KSC SB RAS, Assistant Professor of Experimental Physics and Innovation Technologies department of Siberian Federal University. Research interests: photonic crystals and liquid crystals. E-mail: MaksPyatnov@yandex.ru .

Ivan Vladimirovich Timofeev (b. 1979), Doctor of Physico-Mathematical sciences, Head of Laboratory for Photonics of Molecular Systems, Kirensky Institute of Physics, Federal Research Center KSC SB RAS, Professor of Theoretical Physics and Wave Phenomena department of Siberian Federal University. Research interests: photonic crystals, soft matter, nano- and topological photonics. E-mail: tiv@iph.krasn.ru .

Code of State Categories Scientific and Technical Information (in Russian - GRNTI)): 29.31 .27 Received September 21, 2019. The final version - November 09, 2019. 